\section{Sulfatgele.}

Der Glockerit, der bisher als basisches Eisensulfat betrachtet worden ist und der ungefähr $2 \mathrm{Fe}_{2} \mathrm{O}_{3}+2 \mathrm{SO}_{3}+\mathrm{aq}$ entspricht, ist kolloides Eisenhydroxyd, das Schwefelsäure adsorptionsartig gebunden enthält.

Beim Erhitzen von basischen Eisensulfaten in wässeriger Lösung fällt braunes Eisenoxydhydratgel aus, welches einen hohen Schwefelsäuregehalt besitzt. Bisher wurde dieses Gel als basisches Salz betrachtet. Es liegt aber hier ganz dieselbe Erscheinung vor wie beim Eisenchlorid oder Eisennitrat. Die Entstehung des Glockerits muf in ähnlicher Weise gedacht werden.

\section{Phosphate.}

Eisenoxydphosphate absorbieren Schwefelsăure. Diese Vorgänge kommen in folgenden Formeln zum Ausdruck: 1. 2 $\mathrm{Fe}_{2} \mathrm{O}_{3}+\mathrm{P}_{2} \mathrm{O}_{5}+\mathrm{aq}$ (Delvauxit). 2. $2 \mathrm{Fe}_{2} \mathrm{O}_{3}+\mathrm{P}_{2} \mathrm{O}_{5}+2 \mathrm{SO}_{3}+\mathrm{aq}$ (Diadochit). Aluminiumphosphate (z. B. Türkis, Ceruleolaktin, Henwoodit usw.) adsorbieren $\mathrm{CuO}$, woher ihre blaue Farbe rührt.

\section{Arseniate.}

Der Pittizit, ein Eisenarseniat, adsorbiert in großen Mengen Schwefelsäure.

\section{Silikate.}

1. Chrysokollgruppe. Diejenigen Chrysokolle, welche ungefähr der Formel $\mathrm{CuSiO}_{3}$ entsprechen, enthalten folgende Substanzen adsorbiert: $\mathrm{MgO}, \mathrm{CaO}, \mathrm{ZnO}, \mathrm{PbO}, \mathrm{CO}_{2}, \mathrm{CuCl}_{2}$, $\mathrm{Cu}_{2} \mathrm{Cl}_{2}$. Ob der Demidoffit $\mathrm{P}_{2} \mathrm{O}_{5}(10.22 \mathrm{Proz}$.) adsorptionsartig gebunden enthält, oder $o b$ ein Gemenge vorliegt, muB erst näher untersucht werden. Der Gehalt an $\mathrm{Al}_{2} \mathrm{O}_{3}$ und $\mathrm{Fe}_{2} \mathrm{O}_{3}$ in den Chrysokollen ist mikroskopisch nicht zu erkennen. Hier liegen vielleicht Fällungsprodukte entgegengesetzt geladener Hydrosole vor. Das Kupferpecherz, ein Eisenoxydkupfersilikatgel, enthält öfters $\mathrm{P}_{2} \mathrm{O}_{5}$.

2. Gymnitgruppe. Für diese Gruppe ist namentlich $\mathrm{NiO}$ charakteristisch. Beim
Röttisit, einem Nickelsilikat, wird $\mathrm{As}_{2} \mathrm{O}_{5}, \mathrm{P}_{2} \mathrm{O}_{5}$, $\mathrm{CoO}$ und $\mathrm{CuO}$ durch die Analysen angegeben.

3. Tonerdekieselsäuregele. Hier müssen Produkte der Koagulation scharf geschieden werden von den Verwitterungsgelen, die durch Auslaugung von Silikaten entstanden sind. Die letzteren sind die Kolloide des Ackerbodens, deren Adsorptionsvermögen für Salze namentlich Kalisalze) bekannt ist. Die Koagulationsprodukte (z. B. Allophan, Halloysit) finden sich als Absätze von Grubenwässern. Für die Allophangruppe und Halloysitgruppe sind charakteristisch: $\mathrm{CaO}, \mathrm{MgO}, \mathrm{CuO}, \mathrm{ZnO}, \mathrm{PbO}, \mathrm{CO}_{2}$, $\mathrm{P}_{2} \mathrm{O}_{5}, \mathrm{SO}_{3}, \mathrm{~V}_{2} \mathrm{O}_{5}$ (?).

Für die Gruppe des Montmorillonit $\mathrm{CaO}$, $\mathrm{MgO}, \mathrm{MnO}, \mathrm{Na}_{2} \mathrm{O}, \mathrm{K}_{2} \mathrm{O}$. Für die durch Verwitterung entstandenen Geltone ist namentlich das selektive Adsorptionsvermögen für Kalisalze, ferner ein kaum fehlender Gehalt an $\mathrm{CaO}$ und $\mathrm{MgO}$ erwähnenswert. Viele Geltone (namentlich die Braunkohlentone) enthalten in beträchtlichen Mengen Vanadinsäure. Welche Rolle dieselbe spielt, harrt noch der Aufklärung.

4. Metalloxydkieselsäuregele. Für die Grünerde, ein wasserhaltiges Tonerdeeisenoxydmagnesiasilikat, kommt in Betracht: $\mathrm{Na}_{2} \mathrm{O}$, $\mathrm{K}_{2} \mathrm{O}$, CaO, $\mathrm{MnO}, \mathrm{P}_{2} \mathrm{O}_{5}$ und $\mathrm{CO}_{2}$. Der Chlorophalit enthält $\mathrm{MnO}, \mathrm{CaO}, \mathrm{K}_{2} \mathrm{O}, \mathrm{Na}_{2} \mathrm{O}$. Der Hisingerit, ein wasserhaltiges Eisenoxydsilikat, ist durch einen Gehalt an Schwefelsäure ausgezeichnet. Vielleicht sind mehrere der von dem erstgenannten Verfasser beobachteten sauren Reaktionen ${ }^{4}$ ) an Silikatgelen auf adsorbierte Stoffe zurückzuführen. Es liegt gerade hier ein sehr weites Arbeitsfeld für den Analytiker und Experimentator vor.

\section{Organische Gele.}

Der Dopplerit, im wesentlichen aus Humussäuren bestehend, enthält in mehr oder weniger großen Mengen $\mathrm{CaO}$, weshalb er von Demel als Kalksalz der Humussäuren betrachtet worden ist.

4) Das Referat über die betreffenden Arbeiten von Cornu erscheint demnächst in dieser Zeitschrift.

\title{
Die Systematik der Kolloide des Mineralreiches.
}

\author{
Von F. Cornu, Leoben.
}

Eine Systematik der Kolloide des Mineralreiches muB einerseits den kolloidchemischen Einteilungsgründen gerecht zu werden versuchen, andererseits mub sie aber auch das geologisch- genetische Moment berücksichtigen. Es sei vorausbemerkt, dab die Kolloidchemie auf folgende Gebiete der Mineralogie und Geologie angewendet werden kann und muk. 
1. Auf das Gebiet der Lehre von der Sedimentation in der Natur finden sich in allgemeiner Verbreitung Suspensionen und wahrscheinlich auch Emulsionen. Suspensionen sind z. B. das Gletscherwasser, welches höchst fein verteilte Mineralpartikelchen enthält, ferner das mit Schlammteilchen beladene Wasser aller Bäche und Flüsse und die Suspensionen von Humin- und Ulminsubstanzen in allen Gebieten, in denen Kalkstein oder Dolomit nicht vorkommt. In letzteren werden bekanntlich diese Stoffe durch das Karbonat ausgefällt, weshalb hier die grünblaue Eigenfarbe des Wassers zur Geltung kommt, wie dies z. B. überall in den Kalkalpen der Fall ist. Es liegt nun die Aufgabe vor, die bisherigen Resultate der Physik der Suspensionen auf die entsprechenden Naturvorgänge anzuwenden. Vor allem wäre zu untersuchen, 1. welche Suspensionen kommen in der Natur vor? 2. welche Elektrolyte kommen bei den Pseudofällungen in Betracht? Es müßten bei dieser Untersuchung vor allem die Forschungen Th. Scheerer's, F. Schulze's, Ch. Schloesing's, P. Ebell's, J. Thoulet's, W. H. Brewer's, C. Barus', G. Bodländer's, W. B. Hardy's, W. Spring's, G. Quincke's, H. Bechhold's und A. Müller's berücksichtigt werden. Auch das Vorhandensein von Emulsionen kann in der Natur vorausgesetzt werden, so z. B. bei der Verwesung organischer Substanzen in Sümpfen, ferner in Erdölgebieten, wo man an Emulsionen von Erdölen in Wasser denken könnte. $\mathrm{Da}$ sich noch niemand mit diesem Gebiete speziell befaßt hat, müssen hier zunächst geologische Beobachtungen in größerer Anzahl erfolgen, bevor die Kolloidchemie nutzbringend angewendet werden kann.

2. Auf die Hydrogele und Hydrosole des Mineralreiches. Die groBe Verbreitung der Hydrogele im Mineralreiche, auf welche ich zuerst aufmerksam gemacht habe, läßt eine sehr mannigfaltige Anwendung der kolloidchemischen Forschung zu. Es liegen uns hier zahlreiche Arbeiten von Mineralogen vor, die für die Kolloidchemie von höchstem Werte sind, und die von nun an in fortlaufender Reihenfolge von mir und meinen Mitarbeitern in dieser Zeitschrift referiert werden sollen. Die Hydrosole, durch deren Koagulation die in der Natur vorliegenden Hydrogele teilweise entstanden sind, bedürfen gleichfalls einer sehr eingehenden Untersuchung. Leider hat man sich bisher meist nur mit den vorliegenden Produkten der Koagulation, den Hydrogelen, befaßt. In den meisten Fällen ist die Entstehung dieser Hydrogele noch unbekannt. Es liegt daher hier noch ein ungeheuer weites Arbeitsfeld dem Mineralogen vor Augen. Die unter den Mineralen vorliegenden Hydrogele sind bis auf ein einziges irreversibel. Dies rührt nicht daher, dab es in der Natur keine reversiblen Hydrosole gibt, sondern ist auf den Umstand zurückzuführen, daß noch niemand auf dieses zweite große Gebiet sein Augenmerk gerichtet hat. In allen Grubenwässern kann mit Sicherheit das Vorhandensein reversibler Hydrosole vorausgesetzt werden. Auch das Vorhandensein von Schutzkolloiden in der Natur mub angenommen werden. Leider ermangeln wir hier jeglicher Beobachtungsdaten seitens der Mineralogen. Die allergrößte Anzahl der Hydrogele des Mineralreiches, nämlich die Verwitterungsgele, wozu auch die Hydrogele der Ackererde gehören, sind übrigens gar nicht durch Koagulation entstanden. So z. B. entstehen die Tonerdekieselsäuregele des Ackerbodens, ebenso der Laterit der Tropen durch Auslaugungsvorgänge an Silikaten, die im ersten Falle die Alkalien, im zweiten die Kieselsäure und die Alkalien betreffen.

Es sind Vorgänge ähnlich denen, durch die wir im Laboratorium aus einem Silikate bei der Behandlung mit Säuren Kieselsäuregel erhalten. Bei den Verwitterungsgelen namentlich zeigt sich ein streng gesetzmäbiger Zusammenhang von den klimatischen Verhältnissen, der gleichfalls eines eingehenden Studiums bedarf. Jedem, der den Süden bereist hat, ist die eigentümlich braunrote Farbe des Ackerbodens bekannt, die von wasserärmerem Eisenhydroxydgel herrührt. Bei uns hat das hier wasserreichere Eisenhydroxydgel eine gelbbraune Färbung. In den Tropen findet sich an Stelle der Tone unserer Klimate der brennendrote Laterit, der im wesentlichen aus Aluminiumhydroxydgel und aus Eisenhydroxydgel besteht. Dies gilt für die verbreitetsten Gesteine. Eine gesonderte Untersuchung müBten die Magnesiasilikatgesteine erfahren, die, wie bekannt, stets durch einen abweichenden Pflanzenwuchs gekennzeichnet sind, und denen gewiß verschiedene Verwitterungsprodukte in unseren Klimaten und in den Tropen entsprechen. Diejenige Wissenschaft, die sich von nun ab mit der Verteilung der Hydrogele des Mineralreiches auf die isoklimatischen Zonen unseres Planeten befassen wird und deren Wichtigkeit für die Bodenkunde eine ganz auBerordentiche ist, nenne ich Gelgeographie. Die Gelgeographie ist die Basis der Tier- und Pflanzengeographie. 
3. Auf die hyalinen Körper der Mineralogen, das sind die natürlichen Gläser. Hierauf beziehen sich zwei selbständige Forschungsgebiete. Das erste betrifft die Färbung der natürlichen Gläser. Hierauf muß die Ultramikroskopie angewendet werden. Das zweite Gebiet betrifft die Ausscheidungen in den viskosen Silikatschmelzlösungen, so die Mikrolithen, Sphärolithen u. dgl.

4. Auf die diluten Färbungen von Kristalloiden. Eine grobe Anzahl von Mineralfärbungen ist durch kolloid ausgeschiedene Substanzen hervorgebracht. Berthelot hat die Ansicht ausgesprochen, daß diese Färbungen durch radioaktive Einwirkungen entstanden sind; auch Siedentopf hat dies beim blauen Steinsalz angenommen. Ich schließe mich dieser Ansicht vollinhaltlich an. Wie erfolgreich dieses Arbeitsgebiet ist, kann man bereits aus den grundlegenden Arbeiten von Siedentopf ersehen. Aus meinen Ausführungen dürfte hervorgehen, wieviel Arbeit es hier noch zu leisten gibt. Dabei muB noch berücksichtigt werden, dab beijedem neuen Schritt sich neue Arbeitsgebiete ergeben.

Die in folgendem gegebene Systematik ist in Anbetracht dessen, daB wir am Anfange des Anfangs stehen, noch eine sehr mangelhafte und ist demgemäß von diesem Gesichtspunkte aus zu beurteilen. Hoffentlich kann sie in Kürze durch eine bessere und vollständigere ersetzt werden.
Einteilung der Kolloide des Mineralreiches.

A. I. Suspensionen )

II. Emulsionen $\mathbf{n}\}$ Gebiet der Geologie.

B. Porodine Kolloide (Hydrogele):

I. Irreversible Kolloide

a) juvenile Hydrogele ( $z$. B. Thermalabsätze), Beisp. Plombierit.

b) sekundäre Hydrogele

a) durch Koagulation entstandene

Hydrogele (z. B. Opal):

1. einfache Hydrogele und Adsorptionsverbindungen im Sinne von Cornu, d. h. dem Gesetz der Homoisochemite folgend;

2. irreversible Hydrogele vom Typus des Cassius'schen Purpurs.

b) Verwitterungsgele (z. B. Ton), entstanden durch Auslaugung von Silikaten.

II. Feste Hydrosole (in Grubenwässern, z. B. Ilsemannit).

C. Die kolloiden Färbungen der Gebiet der natürlichen Gläser f Petrographie.

D. Kolloide Färbungen der Kristalloide (bedingt durch Aus- Gebiet der scheidung kolloider Körper, Mineralogie. namentlich durch freie Metalle)

Draga di Moschenizze, 3. April 1909.

\title{
A. Breithaupt, seine Bedeutung für Kolloidchemie und Mineralogie.
}

\author{
von F. Cornu.
}

Es ist bereits jetzt an der Zeit eines hochverdienten Mannes zu gedenken, dessen Forschungen den Ausgang gebildet haben für die Untersuchungen des Verfassers und seiner Mitarbeiter. A. B reith a p t hat im Gegensatze zu den modernen Mineralogen auch die kolloiden Minerale in sein Untersuchungsgebiet einbezogen und hierdurch eine ausgezeichnete Grundlage geschaffen für Mineralogen sowohl, als auch für die Kolloidchemiker. Man hat den Mineralogen der sogenannten naturhistorischen Schule, die bis in die Mitte des neunzehnten Jahrhunderts die herrschende war, den Vorwurf gemacht, daß sie in der Selbstbeschränkung ihr Heil gesehen habe, und auch nicht im Kontakt gestanden sei mit Physik und Chemie.
Dem ist nicht so. Gerade die naturhistorische Schule stand in den vielseitigsten Beziehungen zu den exakten Naturwissenschaften und war immer im Laufenden in bezug auf Entdeckungen sowohl auf physikalischem als auf chemischem Gebiet, was man von den jetzigen Mineralogen gerade nicht in allen Fallen behaupten kann. Haidinger und der ältere Becquerel wendeten bereits zu Farad ay's Zeiten die Elektrochemie auf die Erzlagerstätten an. Bre i th a u p t kannte die Arbeiten Graham's über Kolloide bereits 20 Jahre vor der Entdeckung der Dialyse. Ein weiterer großer Vorteil, den die naturhistorische Schule den moderneren Mineralogen gegenüber voraus hatte, war die Minerognosie, die Uebung im Erkennen der Minerale nach den sogenannten 